\title{
Understanding the Impact of Periodontal Status in Pre \& Post-Menopausal Women with Generalized Chronic Periodontitis and Osteoporosis: A Cross Sectional Study
}

\author{
Sameena Sultana ${ }^{1}$ \\ ${ }^{1}$ Department of Periodontic, College of Dentistry, Jazan University, Jazan, Saudi Arabia \\ Corespondence: Sameena Sultana, Assistant Professor MDS, INDIA Periodontics, Department of periodontic, \\ College of Dentistry, Jazan University, Jazan, Saudi Arabia. Tel: 966-569-133-551. E-mail: \\ dr_sameena_2000@yahoo.com
}

Received: November 7, 2016 Accepted: January 8, 2017 Online Published: February 6, 2017

doi:10.5539/gjhs.v9n4p204 URL: http://dx.doi.org/10.5539/gjhs.v9n4p204

\begin{abstract}
Chronic periodontitis and osteoporosis are two interconnected medical issues, which usually develops complications in the general public. However, public is mostly unaware of their periodontal status. The research study has aimed to understand the impact of periodontal status among pre and post-menopausal women, suffering from periodontitis and osteoporosis. A cross-section approach has been selected for data collection. 70 patients were selected from the outpatient department of SRM dental college in Chennai. SPSS version 20 has been used for statistical analysis. Postmenopausal women have shown statistically significant poor periodontal status as compared to premenopausal women. Similarly, it has also been evaluated that osteoporosis played a progressive role among postmenopausal women for developing periodontitis. The study leads to the conclusion that pre and post-menopausal women must undergo a periodontal assessment on a monthly basis for developing outcomes related to the risk of fractures. Parameters are closely connected with the levels of calcium according to the tests and assessments.
\end{abstract}

Keywords: periodontitis, premenopausal, postmenopausal, osteoporosis, India

\section{Introduction}

\subsection{Introduction to the Problem}

Periodontal disease is considered as an important heath related across the globe. National disease surveillance in regards of World Health Organization has indicated that periodontitis is emerging among the population of USA, UK, Europe, Asia, and other countries at a higher prevalence rate. Periodontium can be characterized as a specialized type of tissues, which are helpful for supporting teeth and maintaining them in the mandibular and maxillary bone. Teeth are surrounded by these issues; therefore, inflammation of such structures is hazardous for the oral cavity. There are four major components, which are directly associated with such dental issues. Four components mainly include gingiva, periodontal ligament, cementum, and alveolar bone. Each of the components has its own architecture, chemical composition, location, function, and biochemical composition (Anil et al., 2013).

Historically, enormous surveys have been conducted across the globe to assess the prevalence rate of periodontal disease in different regions. It has been evaluated that adults are extremely prevalent to this disease, which has made a direct impact on the wellbeing of adults. In United States of America, the prevalence rate of periodontal disease is about 5\%; similarly, this rate is as similar as among Middle Eastern countries. It has been further evaluated that periodontal disease has a direct impact on the oral cavity, and is also associated with enormous hazardous conditions. Periodontitis is a major disease among dentistry domain; therefore, enormous management techniques have been developed in order to counter the complications of this disease (Anil et al., 2013; Armas \& Recker, 2012). Therefore, the healthcare system is working effectively to provide ideal healthcare to the patients, suffering from periodontal disease.

\subsection{Relevant Scholarship}

Osteoporosis can be characterized by increased skeletal fragility along with reduced bone mass, which has mainly 
affected millions of the patients across the globe. It is a chronic condition, which is mainly occurred among the geriatric population. The weakening of the bones is a major symptom, which usually results in fractures, mortality, and disability. It is a fact that the prevalence rate of osteoporosis is continuously increasing because of poor life style. Sedentary life style is considered as a major cause in regards of osteoporosis. However, osteoporosis is itself a major complication of different conditions, which mainly include menopause among women. It is a fact that most of the women usually get involved in osteoporosis as a result of menopause. Due to its chronic state, patients are extremely prone to the condition of fractures (Ashley et al., 2015).

Wactawski-Wende (2001) has indicated that there is a closer association between osteoporosis and periodontal disease among the generalized population. There are enormous characteristics of osteoporosis, which can easily aggravate the complications of the periodontal disease. Periodontitis has been identified as the destruction of soft tissues and alveolar bone, which are essential components of the oral cavity. The study has evidently indicated that osteoporosis is associated with the progression and onset of the periodontal disease among adults. It has been further developed that reduced level of mass density is more likely to be associated with tooth loss and loss of alveolar height. However, less consistency has been identified between the clinical attachment of loss and osteoporosis. The findings of this study have mainly concluded that intense extent of osteoporosis is closely connected with periodontal disease (Dodd \& Rowe, 2013).

Wowern, Klausen \& Kollerup (1994) have also described that osteoporosis is a major risk factor for the periodontal disease among female individuals. The study has validated that attachment of ligaments within oral cavity is closely connected with the severe impact of osteoporosis. Furthermore, the study has also highlighted that age, gender, and smoking are also three major factors, which usually influence the impact of osteoporosis on periodontal disease. The study has revealed that the risk of periodontal disease is much higher among the geriatric population due to osteoporosis as compared to the adult population. Similarly, female patients of osteoporosis have shown much higher involvement rate as compared to the male gender in regards to periodontal disease. Increased extent of smoking has also resulted in increased risk of acquiring periodontal disease due to osteoporosis (Eke et al., 2012).

\subsection{Aim of the Study}

Getting involved with osteoporosis and periodontal disease, the patients are at higher risk of mortality and morbidity. Therefore, it is necessary for the patients to go through periodontal assessment in order to reduce the risk of acquiring complications. Thus, this research study has aimed to analyze the impact of periodontal status among post-menopausal women, suffering from periodontitis and osteoporosis.

\section{Method}

Premenopausal and postmenopausal women were selected for collecting and analyzing data from the outpatient departments of SRM dental college in Chennai. The inclusion criteria for the selection of participants were premenopausal women, aged ranged between 25-65 years and having at least 5 natural teeth in every quadrant. Patients suffering from diabetes, cancer, long-term steroids, and parathyroid disorders have been excluded from the study. On the basis of such criteria, 70 patients have been selected for the study from the outpatient department. The evaluation of periodontal status was done through three ways, which included clinical parameters, biochemical analysis, and radiographic data. Oral hygiene index, periodontal index, and pocket depth attachment loss were done as the clinical parameters. Serum calcium estimation of the patients was also assessed as a part of the biochemical analysis. Mandibular cortex index and composite periodontal index were also assessed through radiographic data. Following criteria were developed for the assessment of periodontal status (Table 1).

Table 1. Oral hygiene Debris index

\begin{tabular}{ll}
\hline Oral hygiene Debris index- simplified (Greene and Vermillion) \\
\hline 0 & $\begin{array}{l}\text { No calculus present } \\
\text { Supragingival calculus covering not more than third of the exposed tooth surface }\end{array}$ \\
\hline 2 & $\begin{array}{l}\text { Supragingival calculus, which covered more than one third of the exposed tooth surface but not more than } \\
\text { two third of it. Similarly, it covered more than one third of the presence of individual flecks of subgingival } \\
\text { calculus, which is about the cervical portion of the tooth. }\end{array}$ \\
& $\begin{array}{l}\text { Supragingival calculus, which covered more than two third of the continuous heavy band of the subvaginal } \\
\text { calculus or the exposed tooth surface around the cervical portion of the tooth. }\end{array}$ \\
\hline
\end{tabular}


The serum has been collected through a proper clinical process. Venus blood was collected from an ante-cubital vein in regards of every participant. The collected blood was centrifuged, and serum was prepared within 2 hours. Serum samples have been stored at $-20^{\circ} \mathrm{C}$. The serum calcium levels have been assessed through calcium reagents. The serum sample was also used for measuring fasting and post-prandial blood sugar level. Furthermore, serum calcium estimation was done through OCPC method. Three test tubes have been taken and were labeled as blank, standard, and test. To each test tube, $0.5 \mathrm{ml}$ of the color reagent along with $0.5 \mathrm{ml}$ of the buffer solution has been added. Afterwards, $0.2 \mathrm{ml}$ of the distilled water has been added to the blank test tube. $0.21 \mathrm{ml}$ of calcium reagent has been added to the standard test tube. All of the solutions have been mixed properly and were kept for 10 minutes. Calorimeter has been used in order to record the readings.

Mendibular cortex has also been assessed through panoramic method. Panoramic radiographs have been obtained from the selected 70 patients. Mandibular cortical shape in regards of dental panoramic radiographs has been determined by observing the mandible from the mental foramen bilaterally. It has been categorized into three groups:

- C1: normal cortex, the endosteal margin of the cortex is even and sharp on both sides

- C2: mildly to moderately eroded cortex, the endosteal margin shows semilunar defects (lacunar resorption) or appears to form endo steal cortical residues

- C3: severely eroded cortex, the cortical layer forms heavy endosteal cortical residues and is clearly porous

Mean and standard deviation were estimated from the sample for each study group (in the case of a continuous variable). Mean values were compared between two study groups by using student's independent t-test. Proportions were estimated for categorical variables and compared between the two study groups by using Pearson's Chi-Square test. In the present study $\mathrm{P}<0.05$ was considered as the level of significance. Three types of tests have been used, which include students' independent t-test, Pearson's chi-square test, and Spearson rank correlation test.

\section{Results}

The results have been analyzed using SPSS version 20.0 and the findings obtained through the analysis have been presented below:

Table 2. Comparison between pre-menopausal and post-menopausal women (Mean and Standard deviation)

\begin{tabular}{lllc}
\hline Variables & $\begin{array}{l}\text { Pre-menopausal } \\
\text { Mean } \pm \text { standard deviation }\end{array}$ & $\begin{array}{l}\text { Post-menopausal } \\
\text { Mean } \pm \text { standard deviation }\end{array}$ & P-value\# \\
\hline OHI-S score & $3.0 \pm 1.3$ & $3.1 \pm 1.0$ & 0.74 (NS) \\
CPITN & $3.0 \pm 0.6$ & $3.7 \pm 0.5$ & $<0.0001$ \\
& & & $<0.0001$ \\
Serum Calcium & $7.0 \pm 1.0$ & $13.3 \pm 1.2$ & (significant) \\
\hline
\end{tabular}

The Mean OHI-S score in Post-menopausal women group (3.1 \pm 1.0$)$ is slightly higher than the pre-menopausal women group (3.0 \pm 1.3$)$. However, there was no significant difference in the Mean of OHI-S scores between the two study groups $(\mathrm{P}=0.74)$. The Mean CPITN in the Post-Menopausal group $(3.7 \pm 0.5)$ was significantly higher than the pre-menopausal group $(3.0 \pm 0.6)(\mathrm{P}<0.0001)$ as shown in table 2. The mean Serum Calcium in Post-Menopausal group (13.3 \pm 1.2$)$ is significantly higher than the Pre-Menopausal group $(7.0 \pm 1.0)(\mathrm{P}=0.0001)$ as shown in Table 4 as shown in Table 2. 
Table 3. Comparison of pre-menopausal and post-menopausal women

\begin{tabular}{|c|c|c|c|c|c|}
\hline \multirow{2}{*}{ OHI-S Grade } & \multicolumn{2}{|l|}{ Pre-menopausal } & \multicolumn{2}{|l|}{ Post-menopausal } & \multirow{2}{*}{ P-Value } \\
\hline & No. of Patients & $\%$ of Patients & No. of Patients & $\%$ of Patients & \\
\hline Good & 01 & $2.9 \%$ & 0 & 0 & 0.42 (Not significant) \\
\hline Fair & 19 & $54.3 \%$ & 16 & $45.7 \%$ & \\
\hline Poor & 15 & $42.9 \%$ & 19 & $54.3 \%$ & \\
\hline \multirow{2}{*}{ CPI index } & \multicolumn{2}{|l|}{ Pre-menopausal } & \multicolumn{2}{|l|}{ Post-menopausal } & \multirow{2}{*}{ P-Value } \\
\hline & No. of Patients & $\%$ of Patients & No. of Patients & $\%$ of Patients & \\
\hline Mild & 26 & $74.3 \%$ & 0 & 0 & $<0.0001$ (significant) \\
\hline Moderate & 9 & $25.7 \%$ & 24 & $68.6 \%$ & \\
\hline Severe & 0 & 0 & 11 & $31.4 \%$ & \\
\hline \multirow{2}{*}{ Type of Periodontitis } & \multicolumn{2}{|l|}{ Pre-menopausal } & \multicolumn{2}{|l|}{ Post-menopausal } & \multirow{2}{*}{ P-Value } \\
\hline & No. of Patients & $\%$ of Patients & No. of Patients & $\%$ of Patients & \\
\hline Mild & 20 & $57.1 \%$ & 0 & 0 & $<0.0001$ (significant) \\
\hline Moderate & 15 & $54.9 \%$ & 14 & $40 \%$ & \\
\hline Severe & 0 & 0 & 21 & $60 \%$ & \\
\hline
\end{tabular}

The distribution of OHI-S grade in a pre-menopausal group with "Good" is $2.9 \%$, fair is $54.3 \%$, and "poor" is $42.9 \%$. In post-menopausal group "Good" is nil, "fair" is $45.7 \%$ and "poor" is $54.3 \%$. However, there was no significant in the distribution of OHI-S between the two study groups $(\mathrm{P}=0.042)$. That is the two study groups are similar as far as OHI-S grades are concerned (Table 3).

The distribution of mandibular cortex index in a pre-menopausal group with "normal" is $35 \%$, "moderate" and "severe" were nil. In post-menopausal group "normal" is nil, "moderate" is $34.30 \%$ and "severe" is $65.70 \%$. The post-menopausal group has shown moderate to severely eroded (unequivocal porosity) mandibular cortex while the pre-menopausal group the mandibular cortex is normal (even and sharp endosteal margins). However, there is the significant difference in the distribution of MCI index scores between the two study groups $(\mathrm{P}<0.001)$. Hence, the two study groups are not similar as far as MCI index is concerned (Table 3).

The distribution of Composite Periodontal Index (CPI) in a Pre-menopausal group with "mild" is $74.30 \%$, "moderate" is $25.70 \%$ and "severe" is nil. In Post-Menopausal group, "mild" is nil, "Moderate" is $68.60 \%$, and "severe" is $31.40 \%$. Hence in the pre-menopausal group the extent of bone loss was mild $(<25 \%)$ to moderate $(25-50 \%)$. And Post-menopausal group exhibited the bone loss from moderate $(25-50 \%)$ to severe $(>50 \%)$. There is a significant difference in the scores between the two study groups $(\mathrm{P}<0.0001)$. That is the two study groups are not similar as far as MCI index is concerned (Table 3).

The distribution of type of periodontitis based on clinical attachment loss in the Pre-Menopausal group is "mild" $57.10 \%$, "moderate" is $42.90 \%$ and "severe" is nil. Post-Menopausal group "mild" is nil, "moderate" is $40 \%$ and "severe" is $60 \%$. In this study, the Pre-menopausal group shows mild to moderate type of periodontitis. While the post-menopausal group shows moderate to severe type of periodontitis. However, there is a significant difference in the distribution of type of periodontitis between the two study groups $(\mathrm{P}<0.0001)$ (Table 3$)$. That is the two study groups are not similar as far as the type of periodontitis based on clinical attachment loss is concerned.

Spearman, rank correlation coefficient test has been used to assess the relation between the two independent variables in each group (Table 4): 
Table 4. Spearman rank Correlation test between different variables in each study group

\begin{tabular}{llllll}
\hline & Group I & & Group II \\
\cline { 2 - 3 } & Correlation coefficient & P-Value & & Correlation coefficient & P-Value \\
\hline CAL \& OHI Score & -.09 & 0.60 & & -0.13 & 0.45 \\
CAL \& MCI & - & - & 0.27 & 0.12 \\
CPITN \& OHI Score & 0.003 & 0.99 & & -0.003 & 0.99 \\
Calcium \& MCI & - & - & & 0.70 & $<0.0001(\mathrm{Sig})$ \\
CPI \& MCI & - & - & & 0.36 & 0.03 (Sig) \\
CPI \& CPITN & 0.46 & $0.006(\mathrm{Sig})$ & 0.46 & $0.006(\mathrm{Sig})$ \\
\hline
\end{tabular}

\subsection{Group 1}

The results have represented the comparison between $\mathrm{CAL}$ and $\mathrm{OHI}$ showing that there is a negative, weak non-significant correlation between these two variables. $\mathrm{P}<0.060$. Comparison between CPITN and OHI score also showed that there is a non-significant correlation between these two variables. $\mathrm{P}<0.99$. Whereas, comparison between CPI and CPITN shows that there is a significant positive correlation between the two variables. $\mathrm{P}=0.006$.

\subsection{Group 2}

The comparison between $\mathrm{CAL}$ and $\mathrm{OHI}$ showed the negative non-significant relation between the variables. $\mathrm{P}=$ 0.45. The CAL and MCI have also been compared, which showed a non-significant correlation between the variables. $\mathrm{P}=0.12$. Comparison between $\mathrm{CPITN}$ and $\mathrm{OHI}$ showed a non-significant negative correlation between the two variables. $\mathrm{P}=0.99$, as the p-value obtained from the analysis is greater than the level of significance. Comparison between serum calcium and MCI further showed a significant correlation between the two variables. P-value $<0.0001$. The comparison between CPI and MCI shows a positive significant correlation between the variables P-value $=0.03$. The comparison between CPI and CPITN shows a significant correlation between the variables. $\mathrm{P}$-value $=0.006$, which is less than the level of significance.

\section{Discussion}

Periodontal disease along with the symptoms and complications of osteoporosis is extremely common among the population within India and across the globe. Both of these chronic medical conditions have significantly common risk factors; therefore, both of these diseases have shown the appropriate extent of effects on the population of elder adults. Postmenopausal osteoporosis has been initiated after surgical or natural menopause, which has resulted in the improved risk of fractures within 15 to 20 years. It is a fact that periodontal examination through different measures must be carried out on the regular basis (Esfahanian et al., 2012). Any sign of osteoporosis must be initially highlighted through such examination in order to reduce the risk of enormous complications. It has been evaluated that changes in clinical attachment and alveolar crestal height are directly associated with the reduced level of bone mineral density among the postmenopausal women (Galal et al., 2014; Gomes-Filho et al., 2013). Therefore, the research study has been carried out to understand the impact of periodontal status.

The relationship between periodontal disease and menopause has not been acknowledged from the mechanisms. Kobayashi et al., (2012) has shown relationship between tooth loss and decreased bone mineral density and deterioration of certain periodontal parameters. There have been several limitations for the use of missing teeth as a surrogated measure of periodontal disease (LaMonte et al., 2013).

Penoni et al. (2015) has referred that tooth loss is considered as a complex phenomenon because it reflects cumulative situations of oral health over period. On the other hand, it is significant to assess that gingival recession might be related with other reasons beyond periodontal disease (Passos et al., 2013). There are no significant differences, which indicated the greater extent of the disease in the periodontal parameters. BMI can be helpful in increasing peripheral conversion of estrogens to provide a protective outcome for measurement. Increased teeth retention, reduction of attachment loss and reduction of gingival inflammation can be observed from numerous studies that suggested a protective role of estrogens in periodontal disease progression (Penoni et al., 2015).

The possible relationship between periodontal association and postmenopausal osteoporosis has been studied in which women with low systematic bone mineral density tended to have greater loss of clinical attachments and alveolar bone (Pepelassi et al., 2012). Hypothesis have been caused for these outcomes, which included less initial bone mineral density, increased production of inflammatory mediators, susceptible genetic factors and destructive 
lifestyle risk factors (Petersen et al., 2005). However, there is not enough evidence to identify the most likely hypothesis. Gomes-Filho et al (2012) has indicated that demonstration of association between periodontal disease and osteoporosis is difficult as both are multifactorial diseases that share multiple risk factors. It has been measured that the major issue that affects the competency to associate osteoporosis with periodontal disease is due to the lack of consistency in diagnosing periodontal disease. The different outcomes have been measured that influence the revelation of periodontal disease, which has determined that periodontal disease is linked with the osteoporosis (Wactawski-Wende et al., 2001).

According to Galal et al (2014), important systematic and oral manifestations have been associated and increased with menopausal period. It has been observed that gingival epithelium becomes atrophic, thinner and more prone to the inflammatory changes. However, there is a reduction in salivary flow rate and salivary composition might be altered that contributes in the development of numerous oral situations (Wowern et al., 1994). Another study indicates that menopause is considered as the main cause of primary osteoporosis that appeared in the sudden reduction in estrogen production and affects jawbones (Passos et al., 2013). It has been recommended that reduction in bone mineral density from estrogen production might contribute to periodontal disease progression. It has been indicated that estrogens also interfere with other periodontal tissues and affect inflammatory responses beside their effect on bone (Melton et al., 2005).

During the current study, the ignorance of the general population regarding the oral health came into light. The study population comprised of the homogenous population with respect to age, race and socioeconomic status. Over 70 female patients were briefed, which were divided as 35 patients of $25-45$ years (premenopausal group) and 35 patients of $46-75$ years (postmenopausal group). During the course of study, it was identified that there was very little knowledge about oral effects of systemic diseases least of all osteoporosis. This could be attributed to the trivial importance of oral health in terms of an orthopedic professional and thus provide very little guidance to the patients. The results of the study have demonstrated that while periodontitis was prevalent in all participants, it was mild to moderate in premenopausal and moderate to severe in postmenopausal subjects. An important finding in the study was the strong correlation between the local factors and the clinical attachment loss and alveolar bone level. The mean Serum Calcium in Post-Menopausal group $(13.3 \pm 1.2)$ is significantly higher than the Pre-Menopausal group $(7.0 \pm 1.0)(\mathrm{P}=0.0001)$. The mandibular cortex index in pre-menopausal group with "normal" is $35 \%$. In post-menopausal group "moderate" is $34.30 \%$ and "severe" is $65.70 \%$. Post-menopausal group has shown a moderate to severely eroded (unequivocal porosity) mandibular cortex while the pre-menopausal group the mandibular cortex is normal.

There is a significant difference in the results of MCI index scores between the two study groups $(\mathrm{P}<0.001)$. Hence the two study groups showed a strong relationship between MCI and osteoporosis is concerned. The type of periodontitis based on clinical attachment loss in the pre-menopausal group is "mild" $57.10 \%$, "moderate" is $42.90 \%$. Post-Menopausal group "moderate" is $40 \%$, and "severe" is $60 \%$. In this study, the Pre-menopausal group shows mild (1-2 $\mathrm{mm}$ of clinical attachment loss) to moderate (3-4mm clinical attachment loss) type of periodontitis. While the post-menopausal group shows moderate $(2-4 \mathrm{~mm}$ CAL loss) too severe $(5 \mathrm{~mm}$ or more CAL loss) type of periodontitis. However, there is a significant difference in the type of periodontitis between the two study groups $(\mathrm{P}<0.0001)$. The study showed positive relationships between the type of periodontitis based on clinical attachment loss and the bone mineral density of the osteoporotic women. The current study had the main limitation of more sophisticated diagnostic aids like DEXA or subtraction radiography, which are more precise for alveolar bone loss. A larger sample size would probably give more information regarding the association between these two entities.

The research has been carried out in order to understand the impact of periodontal status among the premenopausal and postmenopausal women, suffering from osteoporosis and periodontitis. Different tests and assessments have revealed that periodontal parameters are closely connected with the levels of calcium. Therefore, the study has concluded that osteoporosis among postmenopausal women usually plays a major role in the progression of periodontitis. Thus, the postmenopausal patients must go through the proper periodontal assessment on the monthly basis in order to be aware about the probable risk of fractures and other complications. However, no any significant risks have been evaluated in regards of premenopausal patients.

\section{Acknowledgments}

The author is very thankful to all the associated personnel in any reference that contributed in/for the purpose of this research. Further, this research holds no conflict of interest and is not funded through any source. 


\section{Competing Interests Statement}

The authors declare that there is no conflict of interests regarding the publication of this paper.

\section{References}

Anil, S., Preethanath, R. S., AlMoharib, H. S., Kamath, K. P., \& Anand, P. S. (2013). Impact of osteoporosis and its treatment on oral health. The American journal of the medical sciences, 346(5), 396-401. http://dx.doi.org/10.1097/MAJ.0b013e31828983da

Armas, L. A., \& Recker, R. R. (2012). Pathophysiology of osteoporosis: new mechanistic insights. Endocrinology and metabolism clinics of North America, 41(3), 475-486. https://doi.org/10.1016/j.ecl.2012.04.006

Ashley, P., Di Iorio, A., Cole, E., Tanday, A., \& Needleman, I. (2015). Oral health of elite athletes and association with performance: a systematic review. British journal of sports medicine, 49(1), 14-19. https://doi.org/10.1136/bjsports-2014-093617

Dodd, D. Z., \& Rowe, D. J. (2013). The relationship between postmenopausal osteoporosis and periodontal disease. American Dental Hygienists Association, 87(6), 336-344.

Eke, P. I., Dye, B. A., Wei, L., Thornton-Evans, G. O., \& Genco, R. J. (2012). Prevalence of periodontitis in adults in the United States: 2009 and 2010. Journal of dental research, 91(10), 914-920. https://doi.org/10.1177/0022034512457373

Esfahanian, V., Shamami, M. S., \& Shamami, M. S. (2012). Relationship between osteoporosis and periodontal disease: review of the literature. Journal of Dentistry of Tehran University of Medical Sciences, 9(4), 256-64.

Galal, M. A., Zaki, B. M., \& Nassif, N. (2014). The Effect of Omega-3 Fatty Acids on Chronic Periodontitis in Postmenopausal Osteoporotic Females. Life Science Journal, 11(11).

Gomes-Filho, I. S., Oliveira, T. J., Passos, J. S., Cerqueira, E. D. M., da Cruz, S. S., Barreto, M. L., ... \& Sarmento, V. A. (2013). Effect of osteoporosis on periodontal therapy among post-menopausal women. Gerodontology, 30(1), 40-48. https://doi.org/10.1111/j.1741-2358.2012.00643.x

Kobayashi, M., Matsumoto, C., Hirata, M., Tominari, T., Inada, M., \& Miyaura, C. (2012). The correlation between postmenopausal osteoporosis and inflammatory periodontitis regarding bone loss in experimental models. Experimental Animals, 61(2), 183-187. https://doi.org/10.1538/expanim.61.183

LaMonte, M. J., Hovey, K. M., Genco, R. J., Millen, A. E., Trevisan, M., \& Wactawski-Wende, J. (2013). Five-year changes in periodontal disease measures among postmenopausal females: The Buffalo OsteoPerio study. Journal of periodontology, 84(5), 572-584. https://doi.org/10.1902/jop.2012.120137

Melton III, L. J. I., Chrischilles, E. A., Cooper, C., Lane, A. W., \& Riggs, B. L. (1992). How many women have osteoporosis? Journal of bone and mineral research, 7(9), 1005-1010. https://doi.org/10.1002/jbmr.5650070902

Passos, J. S., Vianna, M. I. P., Gomes-Filho, I. S., Cruz, S. S., Barreto, M. L., Adan, L., ... \& Coelho, J. M. F. (2013). Osteoporosis/osteopenia as an independent factor associated with periodontitis in postmenopausal women: a $\begin{array}{lllll}\text { case-control study. } & \text { Osteoporosis international, } & \text { 24(4), }\end{array}$ https://doi.org/10.1007/s00198-012-2130-7

Penoni, D. C., Torres, S. R., Farias, M. L. F., Fernandes, T. M., Luiz, R. R., \& Leão, A. T. T. (2016). Association of osteoporosis and bone medication with the periodontal condition in elderly women. Osteoporosis International, 27(5), 1887-1896. https://doi.org/10.1007/s00198-015-3437-y

Pepelassi, E., Nicopoulou-Karayianni, K., Archontopoulou, A. D., Mitsea, A., Kavadella, A., Tsiklakis, K., \& Horner, K. (2012). The relationship between osteoporosis and periodontitis in women aged 45-70 years. Oral diseases, 18(4), 353-359. https://doi.org/10.1111/j.1601-0825.2011.01881.x

Petersen, P. E., Bourgeois, D., Ogawa, H., Estupinan-Day, S., \& Ndiaye, C. (2005). The global burden of oral diseases and risks to oral health. Bulletin of the World Health Organization, 83(9), 661-669. https://doi.org/10.1111/j.1601-0825.2011.01881.x

Pollycove, R., \& Simon, J. A. (2012). Osteoporosis: screening and treatment in women. Clinical obstetrics and gynecology, 55(3), 681-691. https://doi.org/10.1097/GRF.0b013e31825caa50

Wactawski-Wende, J. (2001). Periodontal diseases and osteoporosis: association and mechanisms. Annals of periodontology, 6(1), 197-208. https://doi.org/10.1902/annals.2001.6.1.197 
Wang, Y., LaMonte, M. J., Hovey, K. M., Mai, X., Tezal, M., Millen, A. E., \& Wactawski-Wende, J. (2015). Association of Serum 17ß-Estradiol Concentration, Hormone Therapy, and Alveolar Crest Height in Postmenopausal Women. Journal of periodontology, 86(4), 595-605. https://doi.org/10.1902/jop.2015.140533

Wowern, N. V., Klausen, B., \& Kollerup, G. (1994). Osteoporosis: a risk factor in periodontal disease. Journal of periodontology, 65(12), 1134-1138. https://doi.org/10.1902/jop.1994.65.12.1134

\section{Copyrights}

Copyright for this article is retained by the author(s), with first publication rights granted to the journal.

This is an open-access article distributed under the terms and conditions of the Creative Commons Attribution license (http://creativecommons.org/licenses/by/4.0/). 